\title{
Miniaturized Bandpass Filter with Wide Stopband Using Complementary Spiral Resonator
}

\author{
Ali K. Horestani ${ }^{1,2}$, Zahra Shaterian ${ }^{1}$, Said Al-Sarawi ${ }^{1}$, Derek Abbott ${ }^{1}$, and Christophe Fumeaux ${ }^{1}$ \\ ${ }^{1}$ School of Electrical and Electronic Engineering \\ The University of Adelaide, Adelaide, SA 5005, Australia \\ ${ }^{2}$ Aerospace Research Institute, Tehran, Iran \\ Emails: \{akarami, zshaterian, alsarawi, abbott, cfumeaux\}@eleceng.adelaide.edu.au
}

\begin{abstract}
In this paper complementary spiral resonators are used in the design of compact planar bandpass filters with wide upper stopband. Based on an inductively loaded transmission line model for the complementary spiral resonators it is shown that the ratio of the spurious resonant frequency to the fundamental resonant frequency can be increased. Comparing two filters realized by conventional open-loop resonators and complementary spiral resonators it is shown that the utilization of complementary spiral resonators not only results in a more compact filter but also that a wide upper stopband is achieved.
\end{abstract}

Index Terms-Complementary spiral resonator, metamaterials, wide stopband, bandpass filter.

\section{INTRODUCTION}

Because of their planar structure and ease of fabrication, conventional microstrip bandpass filters such as endcoupled and parallel-coupled half-wavelength resonator filters are widely used in many microwave applications [1]. However, since the conventional bandpass filters are relatively large in size, large efforts were invested in the miniaturization of these types of filters, for example by using slow-wave or metamaterial inspired structures [2], [3], [4], [5].

Furthermore, while bandpass filters with wide stopband are required in many applications, conventional bandpass filters suffer from unwanted harmonic passbands [6]. To overcome the spurious passband limitation, many approaches have been proposed. For instance, modification of the capacitance and inductance of the coupled line bandpass filters by wiggly-lines have been proposed in [7] and [8] to create a rejection band which suppresses the spurious passband. Utilizing dissimilar resonators such as half- and quarter-wavelength resonators is another approach for spurious passband suppression [9]. Several dissimilar resonators with discriminating coupling can be used to obtain zero coupling coefficient at third harmonic which results in suppressing the third spurious passband and increases the rejection level in the filter's stopband [6]. However, these methods are rather complicated and since they are based on either half- or quarter-wavelength resonators the synthesized filters are relatively large in size. Other approaches such as adding metamaterial particles, e.g. split ring resonators (SRRs) or complementary split ring resonators (CSRRs) to conventional bandpass filters have been used to eliminate the spurious passband [10]. Due to the slow-wave effect of these structures, utilizing these methods also results in more compact filters. Note that in these cases, metamaterial-inspired resonators are not the main building blocks of the filter, they

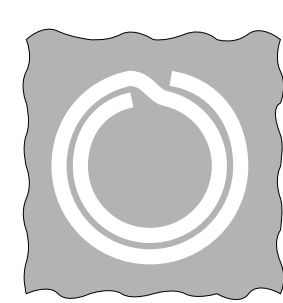

(a)

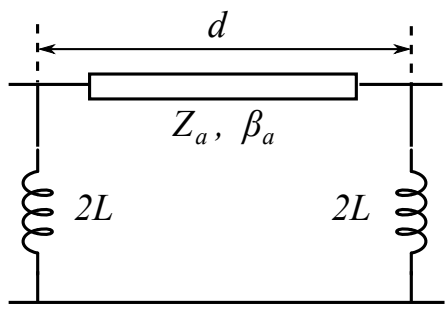

(b)
Fig. 1. (a) Layout of a CSR and (b) inductively loaded transmission line.

are rather used as an extra part to eliminate the harmonic passband [11].

In this paper an alternative approach is used in which complementary spiral resonators (CSRs) are utilized as the central building blocks of a coupled resonator bandpass filter. It is shown that by utilizing the CSR, a coupled resonator bandpass filter can be designed that is not only compact in size but more importantly has a wide stopband.

\section{Complementary Spiral Resonator and Analysis OF SPURIOUS PASSBAND}

One of the widely used methods for designing compact bandpass filters with wide stopband is based on capacitively loaded coupled microstrip open-loop resonators [3]. In the present paper the dual approach is proposed in which CSRs are used as the building blocks of a compact bandpass filter with suppressed spurious passband.

Figure 1 illustrates the geometry and a circuit model of CSR. The model is composed of a TL with characteristic impedance $Z_{a}$, propagation constant $\beta_{a}$ and length $d$, which is loaded with $2 L$ inductors on each end. Following the same approach as for the analysis of the capacitively loaded microstrip line resonators presented in [3] or using the duality theorem, it can be shown that loading the half-wavelength resonator with inductors not only reduces fundamental $\left(f_{0}\right)$ and second harmonic $\left(f_{1}\right)$ resonant frequencies but also results in a higher ratio of the second harmonic resonant frequency to the fundamental resonant frequency, i.e. $f_{1} / f_{0}$. Figure 2 demonstrates the simulated $f_{0}$ and $f_{1}$ of an ideal $\lambda / 2$ resonator (at $2 \mathrm{GHz}$ ), which is loaded with different inductor values. As expected, when $L=0$ the first and second resonant frequencies are at $2 \mathrm{GHz}$ and $4 \mathrm{GHz}$, respectively. However, as the loading inductance is increased both $f_{0}$ and $f_{1}$ are shifted down, thus the resonator's electrical size is decreased. The 


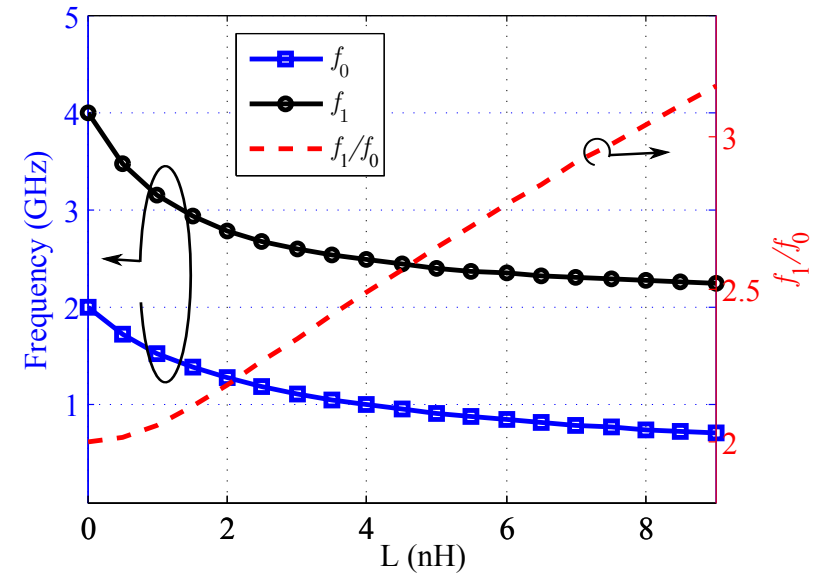

Fig. 2. Simulated fundamental resonant frequency $f_{0}$ and second harmonic resonant frequency $f_{1}$ of an inductively loaded transmission line resonator along with the ratio of $f_{1} / f_{0}$.

figure also depicts the ratio of the second harmonic resonant frequency to the fundamental resonant frequency $f_{1} / f_{0}$ of the structure for different inductance values. The graph clearly shows that even though both resonant frequencies are decreased, the ratio of $f_{1} / f_{0}$ is increased. This property of the CSR can be used in the design of compact coupled resonator bandpass filters with a wide stopband.

\section{Two Pole Wide StopBand Bandpass Filter}

This section provides a comparison between the level of compactness and the bandwidth of the upper stopband in two narrow-band complementary coupled resonator bandpass filters. The first one, shown in Fig. 3, is a two pole filter in which conventional complementary open-loop resonators are used, while the second one, illustrated in Fig. 4, is a similar filter in which CSRs are used as the filter building blocks. The filters are designed to meet the following specifications

\begin{tabular}{lr} 
Center frequency & $2.7 \mathrm{GHz}$ \\
3-dB bandwidth & $200 \mathrm{MHz}$ \\
Passband loss & $3 \mathrm{~dB} \mathrm{Max}$ \\
\hline
\end{tabular}

Given the specification of the filter, the coupling coefficient $M_{12}$ and the external quality factor $Q_{e}$ are obtained from [1]

$$
\begin{aligned}
Q_{e} & =\frac{g_{0} g_{1}}{\mathrm{FBW}}=41 \\
M_{12} & =\frac{\mathrm{FBW}}{\sqrt{g_{1} g_{2}}}=0.058,
\end{aligned}
$$

where $g_{0}, g_{1}$ and $g_{2}$ are the lowpass prototype elements and FBW denotes the fractional bandwidth of the filter. The structure of both bandpass filters and the variables describing the dimensions of the resonators are depicted in Figs. 3 and 4. The structure is realized on a substrate with a relative permittivity of 10.2 and a thickness of $0.78 \mathrm{~mm}$. In the figure, the black lines indicate the $50 \Omega$ feeding microstrip lines on the top of the substrate, whereas the gray shading shows

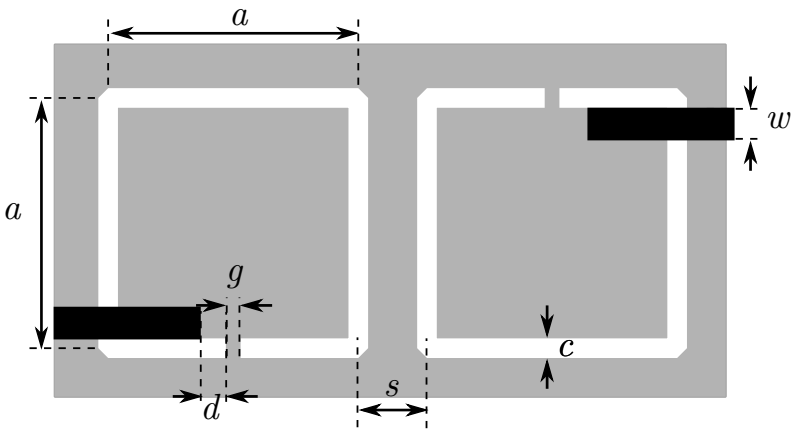

Fig. 3. Structure of a two pole complementary open-loop resonator bandpass filter.

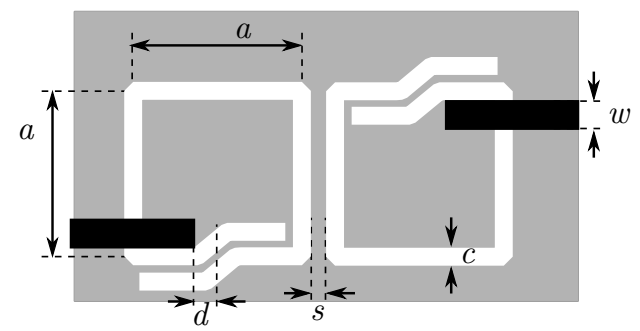

Fig. 4. Structure of a two pole CSR bandpass filter.

the patterning of the ground plane on the bottom side of the substrate.

The next step of the filter design is the characterization of the coupling between the adjacent resonators as well as the external quality factors. The coupling coefficient of the adjacent resonators can be obtained from [1]

$$
M_{i j}=\frac{f_{2}^{2}-f_{1}^{2}}{f_{2}^{2}+f_{1}^{2}}
$$

where $f_{1}$ and $f_{2}$ are the two resonant frequencies of the pair of coupled resonators. The external quality factor $Q_{e}$ can be characterized by [1]

$$
Q_{e}=\frac{2 \pi f_{0} \tau_{11}}{4},
$$

where $f_{0}$ is the resonant frequency and $\tau_{11}$ is the group delay of $S_{11}$.

The electromagnetic simulation tool Agilent Momentum is used for the characterization of the coupling coefficients and the external quality factors. Figure 5 depicts the simulated coupling coefficient of a pair of coupled complementary open-loop resonators (blue solid line) and that of a pair of coupled CSRs (red dashed line) versus the spacing between the resonators in

TABLE I

DIMENSIONS OF COMPLEMENTARY OPEN-LOOP FILTER, SHOWN IN FIG. 3, AS WELL AS THE CSR FILTER, SHOWN IN FIG.4.

\begin{tabular}{|c|c|c|}
\hline Dimension & $\begin{array}{c}\text { Complementary } \\
\text { Open-loop Resonator }\end{array}$ & $\begin{array}{c}\text { Complementary } \\
\text { Spiral Resonator }\end{array}$ \\
\hline$a$ & $6.8 \mathrm{~mm}$ & $4.1 \mathrm{~mm}$ \\
$c$ & $0.4 \mathrm{~mm}$ & $0.4 \mathrm{~mm}$ \\
$g$ & $0.1 \mathrm{~mm}$ & $0.1 \mathrm{~mm}$ \\
$d$ & $0.8 \mathrm{~mm}$ & $0.6 \mathrm{~mm}$ \\
$s$ & $1.6 \mathrm{~mm}$ & $0.4 \mathrm{~mm}$ \\
\hline
\end{tabular}




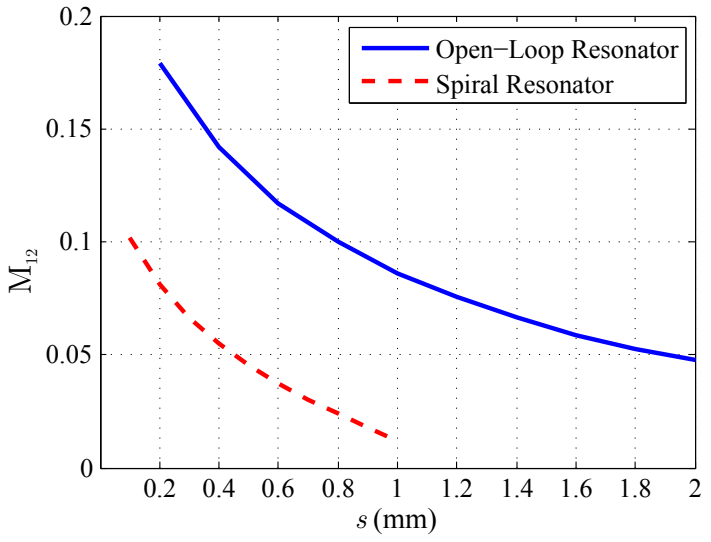

Fig. 5. Simulated coupling coefficient of pairs of complementary open-loop resonators (blue solid line) and that of pairs of CSRs (red dashed line) as a function of the distance between resonators $s$.

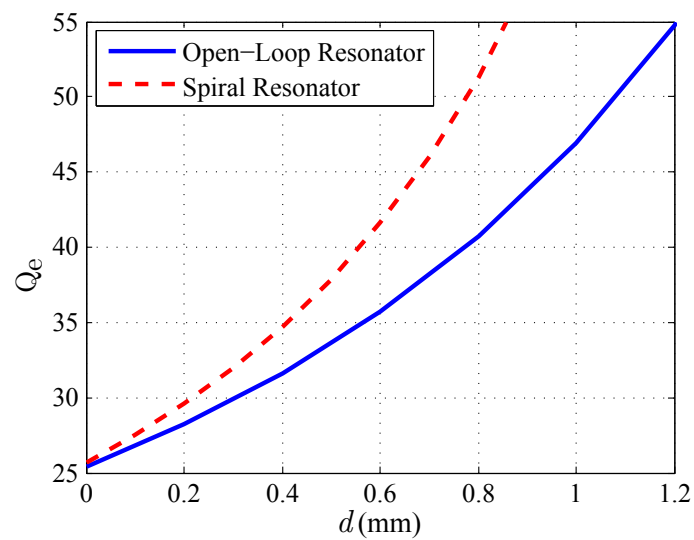

Fig. 6. Simulated external quality factor of the coupling between complementary open-loop resonators (blue solid line) as well as CSRs (red dashed line) with the microstrip feed line as a function of the distance between feed line and symmetry line of the resonators $d$.

each pair. The simulated quality factor of the external coupling between the complementary open-loop resonator as well as the CSR with $50 \Omega$ microstrip line, is depicted in Fig. 6. On the basis of the graphs of the extracted coupling coefficients and external quality factors, two bandpass filters with the given specifications are designed and simulated. Dimensions of the designed filters are given in Table. I.

Figure 7 compares the simulated transmission coefficients of both bandpass filters, showing that both filters meet the specifications, however, the bandpass filter based on CSRs benefits from an $85 \%$ wider upper stopband (defined at $20 \mathrm{~dB}$ rejection). Furthermore, the filter based on the CSR has a more compact size, i.e. a patterned area of $35 \mathrm{~mm}^{2}$ compared to $103 \mathrm{~mm}^{2}$ in the case of the filter based on complementary open-loop resonators.

\section{CONCLUSION}

Using an inductively loaded transmission line model we have shown that an increase in the equivalent inductance characteristic of a CSR results in an increase in the ratio of the second harmonic to the fundamental resonant frequency when compared to a complementary open-loop resonator with

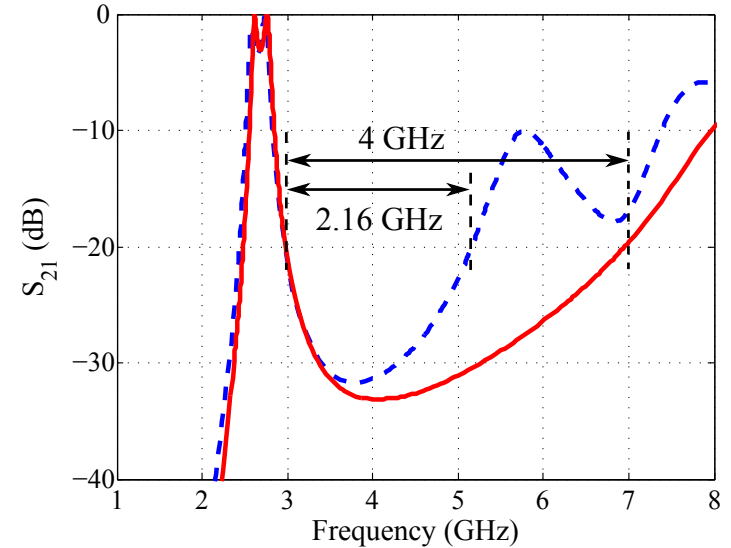

Fig. 7. Comparison between the simulated transmission coefficients of narrowband coupled resonator bandpass filter realized with complementary open-loop resonators (blue dashed line) and that of the same filter realized with CSRs (red solid line).

the same fundamental resonant frequency. This effect has been used to the design of a narrowband coupled resonators filter. Comparison of the CSR filter with an open-loop resonator filter shows that while the proposed filter is $65 \%$ more compact it provides $85 \%$ wider upper stopband.

\section{REFERENCES}

[1] M. J. Hong, J. S. Lancaster, Microstrip Filters for RF/Microwave Applications. Wiley, 2001, ch. 10.

[2] J. García-García, J. Bonache, I. Gil, F. Martín, M. Velazquez-Ahumada, and J. Martel, "Miniaturized microstrip and CPW filters using coupled metamaterial resonators," IEEE Transactions on Microwave Theory and Techniques, vol. 54, no. 6, pp. 2628-2635, 2006.

[3] M. J. L. Jia-Sheng Hong, "Theory and experiment of novel microstrip slow-wave open-loop resonator filters," IEEE Transactions on Microwave Theory and Techniques, vol. 45, no. 12, pp. 2358-2365, 1997.

[4] J. Bonache, I. Gil, J. García-García, and F. Martín, "Novel microstrip bandpass filters based on complementary split-ring resonators," IEEE Transactions on Microwave Theory and Techniques, vol. 54, no. 1, pp. 265-271, 2006.

[5] J. Bonache, F. Martín, F. Falcone, J. García, I. Gil, T. Lopetegi, M. Laso, R. Marqués, F. Medina, and M. Sorolla, "Super compact split ring resonators CPW band pass filters," in IEEE MTT-S International Microwave Symposium Digest, vol. 3, 2004, pp. 1483-1486.

[6] Y. Li, X. Zhang, and Q. Xue, "Bandpass filter using discriminating coupling for extended out-of-band suppression," IEEE Microwave and Wireless Components Letters, vol. 20, no. 7, pp. 369-371, 2010.

[7] T. Lopetegi, M. Laso, F. Falcone, F. Martin, J. Bonache, J. Garcia, L. Perez-Cuevas, M. Sorolla, and M. Guglielmi, "Microstrip wigglyline bandpass filters with multispurious rejection," IEEE Microwave and Wireless Components Letters, vol. 14, no. 11, pp. 531-533, 2004.

[8] T. Lopetegi, M. Laso, J. Hernandez, M. Bacaicoa, D. Benito, M. Garde, M. Sorolla, and M. Guglielmi, "New microstrip wiggly-line filters with spurious passband suppression," IEEE Transactions on Microwave Theory and Techniques, vol. 49, no. 9, pp. 1593-1598, 2001.

[9] S. Lin, Y. Lin, and C. Chen, "Extended-stopband bandpass filter using both half- and quarter-wavelength resonators," IEEE Microwave and Wireless Components Letters, vol. 16, no. 1, pp. 43-45, 2006.

[10] J. García-García, F. Martín, F. Falcone, J. Bonache, I. Gil, T. Lopetegi, M. Laso, M. Sorolla, and R. Marques, "Spurious passband suppression in microstrip coupled line band pass filters by means of split ring resonators," IEEE Microwave and Wireless Components Letters, vol. 14, no. 9, pp. 416-418, 2004.

[11] A. Abdel-Rahman, A. Ali, S. Amari, and A. Omar, "Compact bandpass filters using defected ground structure (DGS) coupled resonators," in IEEE MTT-S International Microwave Symposium Digest, 2005, pp. 1479-1482. 